\title{
CLINICAL PRACTICE GUIDELINES
}

\section{Age Alone is not Adequate to Determine Health-care Resource Allocation During the COVID-19 Pandemic}

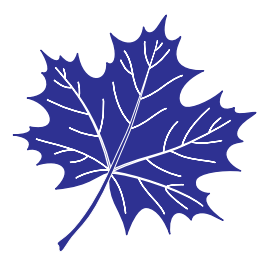

Manuel Montero-Odasso, MD, PhD, FRCPC, AGSF, FGSA ${ }^{1,2,3}$, David B. Hogan, MD, FRCPC FACP ${ }^{4}$, Robert Lam, MD, FCFP ${ }^{5}$, Kenneth Madden, MD, MSc, FRCPC ${ }^{6}$, Christopher MacKnight, MD, MSc, FRCPC ${ }^{7}$, Frank Molnar, MS, MD, FRCPC ${ }^{8}$, Kenneth Rockwood, MD 7 , FRCPC, on behalf the CGS COVID-19 Working Group ${ }^{I}$ Schulich School of Medicine and Dentistry, Department of Medicine and Division of Geriatric Medicine, The University of Western Ontario, London, ON; ${ }^{2}$ Gait and Brain Lab, Parkwood Institute, Lawson Health Research Institute, London, ON; ${ }^{3}$ Department of Epidemiology and Biostatistics, The University of Western Ontario, London, ON; ${ }^{4}$ Division of Geriatric Medicine, Department of Medicine, University of Calgary, Calgary, AB; ${ }^{5}$ Department of Family Medicine, Toronto Western Hospital Family Practice Residency Program, The University of Toronto, Toronto, ON; 'Division of Geriatric Medicine, Department of Medicine, University of British Columbia, Vancouver, BC; ${ }^{7}$ Division of Geriatric Medicine, Department of Medicine, Dalhousie University, Halifax, NS; ${ }^{8}$ Department of Medicine, University of Ottawa, Ottawa, ON, Canada https://doi.org/10.5770/cgj.23.452

\section{ABSTRACT}

\section{Background}

The Canadian Geriatrics Society (CGS) fosters the health and well-being of older Canadians and older adults worldwide. Although severe COVID-19 illness and significant mortality occur across the lifespan, the fatality rate increases with age, especially for people over 65 years of age. The dichotomization of COVID-19 patients by age has been proposed as a way to decide who will receive intensive care admission when critical care unit beds or ventilators are limited. We provide perspectives and evidence why alternative approaches should be used.

\section{Methods}

Practitioners and researchers in geriatric medicine and gerontology have led in the development of alternative approaches to using chronological age as the sole criterion for allocating medical resources. Evidence and ethical based recommendations are provided.

\section{Results}

Age alone should not drive decisions for health-care resource allocation during the COVID-19 pandemic. Decisions on health-care resource allocation should take into consideration the preferences of the patient and their goals of care, as well as patient factors like the Clinical Frailty Scale score based on their status two weeks before the onset of symptoms.

\section{Conclusions}

Age alone does not accurately capture the variability of functional capacities and physiological reserve seen in older adults. A threshold of 5 or greater on the Clinical Frailty Scale is recommended if this scale is utilized in helping to decide on access to limited health-care resources such as admission to a critical care unit and/or intubation during the COVID-19 pandemic.

Key words: aged, frailty, COVID-19, health-care resources, mechanical ventilation.

\section{"Rashness belongs to youth; prudence to old age." -Marcus Tullius Cicero in "De Senectute", 44 BC}

As encapsulated by Cicero's quote from "De Senectute", aging comes with wisdom and prudence. Here we argue that, in times of extreme turmoil and difficulty, prudence and wisdom should prevail while making critical decisions.

One goal of the Canadian Geriatrics Society (CGS) is to foster the health and well-being of older Canadians and older adults worldwide. Although severe COVID-19 illness and significant mortality occur across the lifespan, the fatality rate increases with age, especially for people over 70 years of age. ${ }^{(1)}$

During the last few weeks, the emerging discussion about the rationing of health-care resource utilization has been tainted with ageism. ${ }^{(2,3)}$ The dichotomization of COVID-19 patients by age has been brought up as a way to decide who 
will get mechanical ventilation when critical care unit beds and ventilators are limited. In geriatrics and gerontology, alternative approaches to using chronological age have long been advocated in making these decisions. There is a need to look at the treatment choices and prognosis of the individual, whatever their age. In Italy, where the health-care system has faced incredible strain from the COVID-19, age has been proposed as a criteria for making these decisions. ${ }^{(4)}$ Other countries have promoted using frailty. For example in the UK, the updated NICE guidelines for the allocation of critical care interventions suggests using the 9-point Clinical Frailty Scale (CFS) to assess frailty status, and to use this information to help make these decisions. ${ }^{(5,6)}$

At the CGS, we strongly believe that rationing critical care admission and/or life-saving ventilation solely on the basis of age is not acceptable. While an established body of evidence supports that mechanical ventilation would likely be futile in some ill frail older adults with multiple morbidities, there is a critical ethical difference between decisions made on the basis of futility versus those based on age. (7)

\section{CGS HEALTH-CARE RESOURCE UTILIZATION RECOMMENDATIONS}

1. Age alone should not drive decisions for health-care resource allocation.

Instead, the wishes of the person (including as expressed in an advance directive if the person is unable to speak for themselves at the time), the presence and degree of frailty, and other aspects of their health before the onset of the acute illness should be considered. The British Medical Association guidance on ethical issues with COVID-19, ${ }^{(8)}$ notes an association between older age and mortality, but it is still unknown whether this reflects an actual effect of age or a correlation between age and co-morbidities that in themselves effect survival rates. We strongly believe that decisions must not be based solely on chronological age. Ethical triage requires identification of clinically relevant facts about individual patients and their likelihood of benefiting from possible medical interventions. Younger patients should not be automatically prioritized over older ones.

2. Decisions on health-care resource allocation should take into consideration the preferences of the patient and their goals of care.

Resource allocation protocols should treat older persons in a similar fashion to younger persons. Patients' wishes for guiding their care ought to be clarified in advance of a critical illness. Older adults have higher prevalence of mild cognitive impairment and comorbidities that can transitory exacerbate when they are ill, and consequently, they may not be able to state their goals of care while very sick. Available data show that less than a fifth of Canadians have an advance care plan. $(9,10)$ These can be challenging conversations, but older adults should be encouraged to express their wishes to their family and document their goal of care prior to becoming severely ill (https://www.advancecareplanning.ca/). ${ }^{(11)}$

3. Decisions on access to critical care or mechanical ventilation for older adults should be individualized.

Older adults' capability and functionality are not tightly tied to their chronological age. Frailty has helped geriatricians and researchers on aging to understand and grasp the variability seen in older patients, including in the critical care setting. ${ }^{(12)}$ Evidence shows that frailty status determined by a simple instrument such as the CFS is useful in identifying older adults at increased risk of adverse events, morbidity, and mortality in critical care units. ${ }^{(13)}$ The identification of frailty and its severity could improve prognostication, and identify those more likely to benefit and respond to invasive treatments. We advocate for an approach similar to the NICE's rapid guideline for deciding on the appropriateness of critical care with a COVID-19 infection (https://www.nice.org.uk/guidance/ng159/resources/ critical-care-admission-algorithm-pdf-8708948893).

4. If used to help make decisions about access to limited health-care resources, the CFS score should be based on the person's status two weeks before the onset of COVID symptoms and not how they present at the moment.

The use of the CFS by untrained or minimally trained clinicians can lead to inaccurate scoring, especially if the assigned score is based on the person's status the day of their presentation. Online training modules on how to administer the CFS are available at the following link: https://rise.articulate.com/share/deb4rT021vONbq4AfcMNRUudcd6QMts3\#/

Where available, a consultation with a geriatrician or health-care worker who specializes in the care of older patients should be procured for required assistance on how to appropriately employ the CFS.

5. If used to help make decisions about access to limited health-care resources such as admission to a critical care unit and/or intubation during the COVID-19 pandemic, a CFS score of 5 or greater should be used.

A critical care study done in Canada where the CFS was utilized that enrolled over 1,200 older adults found that the risk of mortality rose with increasing frailty severity. ${ }^{(13)} \mathrm{As}$ shown in Figure 1 though, older adults with CFS $\geq 5$ still have one-year survivals of roughly $50 \%$. This provides a rationale for using a score of 5 or greater to help inform these decisions, but underscores the need to consider other factors, as well. Please note that decisions about the need for hospitalization should be considered separately.

\section{CONCLUDING REMARKS}

We recognize that, for many of us, the COVID-19 pandemic is our first public health emergency. In these circumstances, instead of ethics optimized for autonomy, as is the usual case, 


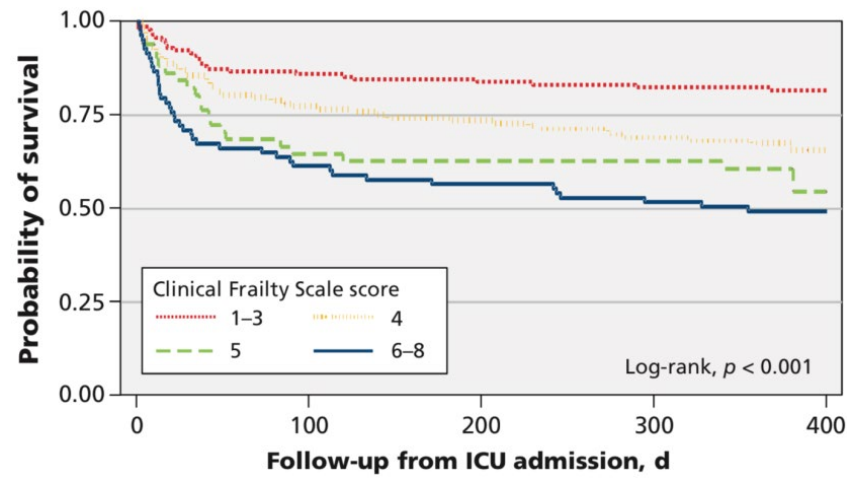

FIGURE 1. Probability of survival of critically ill older adults with different Clinical Frailty Scale scores, since admission to an Intensive Care Unit. Reproduced with permission from Bagshaw et al. 2014(13)

ethical decisions must now optimize for benefit. In general, these calculations will favour younger people over older people. Even so, the potential for benefit does not automatically map to age. Understanding this will be crucial for older adults, where heterogeneity of outcomes is the greatest. Being able to recognize in this group those who have potential for benefit is a reasonable requirement for guidelines developed to deal with COVID-19 infections.

\section{KEY POINTS}

1. Age alone should not drive decisions for health-care resource allocation.

2. Decisions on health-care resource allocation should take into consideration the preferences of the patient and their goals of care.

3. Decisions on access to critical care or mechanical ventilation for older adults should be individualized.

4. If used to help make decisions about access to limited health-care resources, the CFS score should be based on the person's status two weeks before the onset of COVID symptoms, and not how they present at the moment.

5. If used to help make decisions about access to limited health-care resources such as admission to a critical care unit and/or intubation during the COVID-19 pandemic, a CFS score of 5 or greater should be used.

\section{ACKNOWLEDGEMENTS}

The authors would like to thanks CGS COVID-19 Working Group members who provided in-put to this article: Drs Nathan Stall, Olga Theou, and Camilla Wong.

\section{CONFLICT OF INTEREST DISCLOSURES}

MMO is a member of the Board of directors, SecretaryTreasurer, and co-Chair of the COVID-19 Working Group of the Canadian Geriatrics Society. DBH has none to declare.
FM is CGS past president and co-chair of the Covid-19 Working Group of the Canadian Geriatrics Society. KM is editor-in-chief of the Canadian Geriatrics Journal. KR has asserted copyright of the Clinical Frailty Scale; use of the scale is free for research, educational, and not-for-profit health-care use.

\section{REFERENCES}

1. Onder G, Rezza G, Brusaferro S. Viewpoint: Case-fatality rate and characteristics of patients dying in relation to COVID-19 in Italy [Internet]. JAMA. 2020:4688.

2. Aronson L. Ageism is making the pandemic worse [Internet]. The Atlantic. 2020, March 28. Available from: https://www. theatlantic.com/culture/archive/2020/03/americas-ageismcrisis-is-helping-the-coronavirus/608905/

3. Pittis D. Politicians who consider sacrificing the old for the sake of the economy face a backlash [Internet]. CBC News. 2020 Mar 26. Available from: https://www.cbc.ca/news/business/ coronavirus-covid-economy-seniors-1.5510079

4. Vergano M, Bertolini G, Giannini A. Clinical ethics recommendations for the allocation of intensive care treatments, in exceptional, resource-limited circumstances [Internet]. It Soc Anesthesia, Analgesia, Resuscitation Inten Care (SIAARTI). 2020 Mar 16.

5. Rockwood K, Song X, MacKnight C, Bergman H, Hogan DB, McDowell I, et al. A global clinical measure of fitness and frailty in elderly people. CMAJ. 2005;173(5):489-95.

6. Juma S, Taabazuing MM, Montero-Odasso M. Clinical Frailty Scale in an acute medicine unit: a simple tool that predicts length of stay. Can Geriatr J. 2016;19(2):34-39.

7. Jecker NS, Schneiderman LJ. Futility and rationing. Am J Med. 1992;92(2):189-96.

8. British Medical Association (BMA). COVID-19-Ethical issues. A guidance note [Internet]. London, UK: BMA; 2020. Available from: https://www.bma.org.uk/media/2226/bmacovid-19-ethics-guidance.pdf

9. Heyland DK, Barwich D, Pichora D, Dodek P, Lamontagne F, You JJ, et al. Failure to engage hospitalized elderly patients and their families in advance care planning. JAMA Intern Med. 2013;173(9):778-87.

10. Advance Care Planning in Canada. National Poll (2019) Attitudes and Behaviours. 'Speak Up' Canada [Internet]. Ottawa, ON: Canadian Hospice Palliative Care Assoc; 2019. Available from: https://www.advancecareplanning.ca/resource/national-poll-info-graphic/?utm_source $=$ cnw\&utm medium $=$ referral\&utm_campaign $=$ poll1 $\overline{9}$

11. Advance Care Planning in Canada. COVID-19 Resources for health care professionals. 'Speak Up' Canada [Internet]. [n.d.]. Available from: https://www.advancecareplanning.ca

12. McDermid RC, Stelfox HT, Bagshaw SM. Frailty in the critically ill: a novel concept. Crit Care. 2011;15(1):301.

13. Bagshaw SM, Stelfox HT, McDermid RC, Rolfson DB, Tsuyuki RT, Baig N, et al. Association between frailty and short- and long-term outcomes among critically ill patients: a multicentre prospective cohort study. CMAJ. 2014;186(2):E95-E102.

Correspondence to: Manuel Montero-Odasso, MD, PhD, FRCPC, AGSF, FGSA, Schulich School of Medicine and Dentistry, The University of Western Ontario, 550 Wellington Rd., Room A3-116, London, ON N6C 0A7, Canada

E-mail: mmontero@uwo.ca 